\title{
Intraoperative electron radiation therapy as an important treatment modality in retroperitoneal sarcoma
}

\author{
Raeshell S. Sweeting, MD ${ }^{a}$, Allison M. Deal, MS ${ }^{b, c}$, Omar H. Llaguna, MD ${ }^{a}$, Brian K. \\ Bednarski, MD ${ }^{a}$, Michael O. Meyers, MD ${ }^{a, c}$, Jen Jen Yeh, MD ${ }^{a, c}$, Benjamin F. Calvo, MD ${ }^{a, c}$, \\ Joel E. Tepper, MD ${ }^{c, d}$, and Hong Jin Kim, MD ${ }^{a, c,{ }^{*}}$ \\ aDivision of Surgical Oncology, University of North Carolina School of Medicine at Chapel Hill, \\ Chapel Hill, North Carolina \\ bBiostatistics Core Facility, University of North Carolina School of Medicine at Chapel Hill, Chapel \\ Hill, North Carolina \\ 'The Lineberger Comprehensive Cancer Center, University of North Carolina School of Medicine \\ at Chapel Hill, Chapel Hill, North Carolina \\ ${ }^{\mathrm{d} D e p a r t m e n t}$ of Radiation Oncology, University of North Carolina School of Medicine at Chapel \\ Hill, Chapel Hill, North Carolina
}

\begin{abstract}
Background-Local recurrence (LR) rates in patients with retroperitoneal sarcoma (RPS) are high, ranging from $40 \%$ to $80 \%$, with no definitive studies describing the best way to administer radiation. Intraoperative electron beam radiation therapy (IOERT) provides a theoretical advantage for access to the tumor bed with reduced toxicity to surrounding structures. The goal of this study was to evaluate the role of IOERT in high-risk patients.
\end{abstract}

Methods-An institutional review board approved, single institution sarcoma database was queried to identify patients who received IOERT for treatment of RPS from 2/2001 to 1/2009. Data were analyzed using the Kaplan-Meier method, Cox regression, and Fisher Exact tests.

Results-Eighteen patients (median age 51 y, 25-76 y) underwent tumor resection with IOERT (median dose $1250 \mathrm{cGy})$ for primary $(n=13)$ and recurrent $(n=5)$ RPS. Seventeen patients received neoadjuvant radiotherapy. Eight high-grade and 10 low-grade tumors were identified. Median tumor size was $15 \mathrm{~cm}$. Four patients died and two in the perioperative period. Median follow-up of survivors was $3.6 \mathrm{y}$. Five patients (31\%) developed an LR in the irradiated field. Three patients with primary disease (25\%) and two (50\%) with recurrent disease developed an LR $(P=0.5)$. Four patients with high-grade tumors $(57 \%)$ and one with a low-grade tumor $(11 \%)$ developed an LR $(P=0.1)$. The 2- and 5-y OS rates were $100 \%$ and $72 \%$. Two- and 5-y LR rates were $13 \%$ and $36 \%$.

(C) 2013 Elsevier Inc. All rights reserved.

* Corresponding author. Division of Surgical Oncology, University of North Carolina School of Medicine, P1150 POB, CB \#7213, 170 Manning Drive, Chapel Hill, NC 27599-7213. Tel.: +1 919966 5221; fax +1 919966 8806. kimhj@ med.unc.edu (H.J. Kim).

No financial disclosures. 
Conclusions-Using a multidisciplinary approach, we have achieved low LR rates in our highrisk patient population indicating that IOERT may play an important role in managing these patients.

\section{Keywords}

Sarcoma; Retroperitoneal sarcoma; Intraoperative radiation

\section{Introduction}

Soft tissue sarcomas are rare tumors and represent only $1 \%$ of cancers diagnosed in the US for an annual incidence of 11,280 cases [1]. Retroperitoneal sarcomas (RPS) represent approximately $12 \%-15 \%$ of all sarcomas. Because of the rarity of this tumor type, there is little definitive data in the literature regarding management. The gold standard of treatment remains surgical resection with negative margins. However, given the location of these tumors and the often large size at the time of presentation, it is difficult to achieve wide margins at the time of surgery. As a result, the benefit of a multidisciplinary approach to determine which patients are at a high risk is essential. Local recurrence (LR) rates for RPS can range from $40 \%$ to $80 \%$ with 5 y overall survival (OS) historically at 50\% [2-8]. Seventy-five percent of deaths in patients with RPS are due to LR $[9,10]$. Radiation therapy in conjunction with surgery has been shown to reduce rates of LR in high-risk sarcomas, particularly in extremity sarcoma [11]. However, the delivery of radiation in patients with RPS presents a clinical dilemma. In extremity soft tissue sarcomas, external beam radiation therapy (EBRT) at doses of $>6400$ cGy can be administered with minimal toxicity or damage to surrounding structures and negative margins can usually be obtained [10]. However, due to the deep nature of the tumor, these parameters are not easily accomplished when treating RPS.

RPS arise in close proximity to vital organs such as bowel, kidney, and liver. It is often difficult or impossible to deliver effective doses of EBRT to the entire tumor bed without significant toxicity to these surrounding structures. As a result, IOERT provides a theoretical advantage over EBRT in treating these tumors. IOERT allow for sufficient delivery of radiation directly to the tumor bed while placing vital organs outside the field of radiation to reduce toxicity. Additionally, IOERT can provide a boost of radiation therapy to areas that would otherwise be difficult to control with surgery and EBRT. IOERT is also useful in patients who have previously received EBRT present with a recurrence. Despite these theoretical advantages, there are no clear indications in the literature for when IOERT is most beneficial.

Few studies have been able to evaluate the benefits of IOERT use in patients with high-risk RPS due to the rarity of this tumor type. Given the potential advantages of incorporating IOERT into the management of patients with RPS, we sought to analyze our experience to add to the existing body of research and improve the treatment of this locally aggressive disease. We hope to provide further information on the risk of local regional failure after resection and IOERT. 


\section{Methods}

Patients were selected from a single institution, prospective database that has been approved by our institutional review board. Patients were identified for the sarcoma database using operative case logs. Hospital medical records were reviewed for clinic notes, radiology reports, operative notes, and pathology reports. In addition, social security death records were reviewed for each patient. All patients who underwent IOERT for treatment of either primary or recurrent RPS from April 2002 to January 2009 were included in this study, for a total of 18 patients.

Radiation was administered intraoperatively via a Mobetron device (IntraOp Medical, Sunnyvale, CA) in a dedicated OR staffed for IOERT administration. Intraoperative radiation doses ranged from 1000 to $2000 \mathrm{cGy}$. Most patients also received neoadjuvant radiation therapy to a dose of approximately $4500 \mathrm{cGy}$. Radiation doses varied depending on whether the tumor was being treated initially or there had been radiation delivered previously and whether there was pathologic or clinical evidence of positive margins. Patients who had not been previously irradiated generally received preoperative EBRT to a dose of $45 \mathrm{~Gy}$ ( $4500 \mathrm{cGy}$ ) over $5 \mathrm{wk}$. At the time of surgery, if they received preoperative radiation therapy and there was no evidence of a positive margin but the patient was thought to still be at risk of local failure because of the infiltrative nature of the tumor, an additional $10 \mathrm{~Gy}$ was used postoperatively. If there was greater concern for margin positivity, then a dose of $1500 \mathrm{cGy}$ was generally used. For patients who did not receive EBRT, a dose of $12.5-15$ cGy was used for negative margins and a dose up to $20 \mathrm{~Gy}$ for positive margins or known residual tumor.

Four surgical oncologists performed the surgical procedures. A tumor board consisting of surgical oncologists, radiation oncologists, surgical pathologists, and medical oncologists collaboratively made the decision for IOERT. Although there were no set criteria for recommending that a patient receive IOERT, the patients selected for this approach were those thought to be at high-risk patients for LR due to factors such as high grade, large tumor size, or proximity to vital organs.

\subsection{Statistical methods}

Percentages of development of LR were compared between groups using Fisher Exact test, and the Kaplan-Meier method was used to compare OS and time to LR between categories. Cox regression was used to estimate the association between tumor size and OS and time to LR. OS was calculated as the time from operation at UNC when IOERT was delivered to the date of death or last follow-up. Time to LR was calculated as the time from operation to the date of LR or last follow-up. The upper bound of the $95 \%$ confidence interval (CI) for median time to LR could not be estimated due to censoring.

\section{Results}

A total of 18 patients were included in the study. Four patients died and two deaths were perioperative and the other two patients died of disease. The median follow-up of survivors was $43 \mathrm{mo}$ (range 3-98 mo), and all but one patient had >1 y of follow-up. Of the 18 
patients included in the study, $13(72 \%)$ had primary disease and five (28\%) presented with recurrent disease. There were an equal number of males and females. Eleven patients (61\%) were white and seven patients were black (39\%). The median age was 51 y (range 25-76 y).

Half of the tumors were liposarcomas, five (28\%) were leiomyosarcomas, two were malignant fibrous histiocytoma (11\%), and there was one sarcomatoid carcinoma and one tumor that could not be classified into a specific histologic type. Eight of the tumors were high grade and 10 were low grade. Median tumor size was $15 \mathrm{~cm}$ (range 3-41 cm). Margins were R0 in $16(89 \%)$ and R1 in $2(11 \%)$ (Table 1).

All 18 patients received IOERT with a median dose of 1250 cGy (range 1000-2000). Seventeen of these patients also received neoadjuvant radiotherapy with a median dose of 4500 cGy (range 4500-5040).

Postoperative complications included an anastomotic leak $(n=1)$, a chyle leak $(n=1)$, and a retroperitoneal abscess $(n=1)$.

Sixteen of the 18 patients were available for analysis of LR as a result of the two perioperative deaths. Five patients (31\%) developed an LR, none of which were in the setting of distant metastasis. Three of the evaluable patients (25\%) with primary disease and two of the evaluable patients (50\%) with recurrence disease experienced an LR $(P=0.5)$. Four patients with high-grade tumors $(57 \%)$ and one patient with low-grade tumors (11\%) had an LR $(P=0.1)$ (Table 2). Median time to LR was 6.08 y (95\% CI: 2.37). The 2- and 5y LR free rates were $87 \%$ (95\% CI: $56.4 \%-96.5 \%$ ) and 64\% (95\% CI: $27.8 \%-85.8 \%$ ). No significant differences were noted in time to LR in patients with primary versus recurrent disease $(P=0.7)$, high-versus low-grade lesions $(P=0.2)$, multivisceral resection $(P=$ $0.18)$, or larger tumor size at presentation $(P=0.6)$ (Fig. A). Three of the five patients with LR underwent re-resection and had no evidence of disease at the time of the study. The other two patients died of disease approximately 2 and 3 y later.

The 2- and 5-y OS rates were 100\% and 72\% (95\% CI: 23.4\%-92.8\%). No significant differences were noted in OS in patients with primary versus recurrent disease $(P=0.3)$, high-versus low-grade lesions $(P=0.8)$, multivisceral resection $(P=0.5)$, or larger tumor size at presentation $(P=0.9)$ (Fig. 1B).

\section{Discussion}

Our study examines the recurrence patterns and OS of 18 RPS patients treated with IOERT. Our results support existing data that IOERT does play an important role in managing patients with high-risk RPS. In this high-risk patient population, our LR rate of $31 \%$ and $5-y$ OS of $72 \%$ are quite favorable. There have been a small number of historical studies examining outcomes in patients with RPS, which convey the aggressive nature of this disease consistent with our findings [4,12-15] (Table 3). Due to the small size of our study, we were not able to perform multivariate analysis to determine specific factors associated with recurrence and OS in the setting of IOERT. However, we saw no statistically significant difference in time to LR or OS when comparing high-versus low-grade lesions, primary versus recurrent disease, large tumor size or multivisceral resection, suggesting that 
the LR and OS benefits can be achieved by all these subgroups in carefully selected populations.

There are a small number of studies exploring the use of IOERT in patients with RPS. These studies have found similar recurrence rates to our study [3,10,16-18] (Table 4). In the study of 28 patients by Yoon et al., the importance of negative margins is again discussed as the best method of controlling disease. Twelve of the 28 patients in their study received IOERT in cases of close or positive posterior margins and they postulate that IOERT may be an important tool in helping to sterilize posterior margins and margins that would otherwise be hard to access with surgery alone. In the study by Geischen et al., a comparison is made between patients receiving neoadjuvant radiation and surgery alone, to neoadjuvant radiation plus IOERT. A total of 37 patients are included in their study, 20 of whom received IOERT. In comparing those patients receiving IOERT with patients who did not, the authors demonstrate a clear improvement in local control and OS in patients receiving IOERT in their carefully selected patient population with 5-y OS of 74\% in the IOERT group compared with $30 \%$ in the neoadjuvant alone group. Consistent with these findings, our 5-y OS rate of $72 \%$ is substantially improved over historical rates around $50 \%$.

IOERT can be administered with a complication profile similar to that of EBRT. Some studies report increased neurotoxicity in patients receiving IOERT due to the proximity of the administered dose to neurovascular bundles [5,19-21]. We report on all postoperative complications but do not elaborate on radiation-specific complications. However, an earlier study at our institution by Caudle et al. reported that complication rates with neoadjuvant radiotherapy and IOERT were comparable to complications with neoadjuvant radiation therapy alone. In this study, five of the 14 patients studied received IOERT in addition to neoadjuvant radiation therapy with no difference in side effect profiles [22]. This is consistent with more recent studies suggesting that more favorable toxicity profiles with preservation of recurrence and survival benefits can be achieved at lower accepted doses of IOERT [23].

The limitations of this study are that it is a single institution, retrospective analysis and like most studies of treatment modalities in RPS, there are a small number of patients. Despite these limitations, we have been able to provide valuable data regarding our experience with IOERT in this rare and challenging patient population. The results of our study are easily translatable to current patient populations in that none of our patients received chemotherapy, virtually all received EBRT preoperatively, and IOERT was administered at low relative doses.

In our study, we did not use specific criteria to determining that a patient is an appropriate candidate for IOERT. However, there are some characteristics that are associated with a high risk of LR. Patients with high-grade tumors and large tumor size are at greater risk for recurrence. Specific to IOERT as a modality, patients with tumors that closely abut vital structures, particularly neurovascular structures, are potential candidates for IOERT. Finally, patients who have already received radiation can have IOERT as a boost of radiation in a previously irradiated field. Ultimately, we use a multidisciplinary approach to decision making to make a final recommendation for IOERT. 
Our results indicate that improved LR rates can be achieved using multimodality treatment including IOERT in patients with high-risk RPS. More robust studies are needed to better characterize the advantages of IOERT and to further delineate which patients receive the greatest benefit from this treatment modality. Previous attempts at randomized controlled trials of IOERT have been limited by difficulty in accruing a substantial patient population given the rarity of this tumor type. The one small randomized controlled trial of 35 patients conducted in 1993 by Sindelar et al. found an improved incidence of LR with use of intraoperative radiation therapy (IORT) $(n=15)$ versus postoperative EBRT alone with six of 16 patients receiving IORT experiencing an LR compared with 16 of the 20 patients who received EBRT alone. However, some patients in this trial received chemotherapy, which is currently not the standard of care and IORT doses were higher than currently accepted doses resulting in higher rates of neuropathy [5].

In tumors such as RPS in which local failure is the primary contributor to disease morbidity and mortality, improved methods of local control are of great importance. Results from our study and review of the literature support the idea that IOERT is indeed a powerful tool in sterilizing local margins and improving local control and therefore disease-associated mortality.

\section{REFERENCES}

1. American Cancer Society. Cancer facts and figures 2012. 2012

2. Catton CN, O'Sullivan B, Kotwall C, Cummings B, Hao Y, Fornasier V. Outcome and prognosis in retroperitoneal soft tissue sarcoma. Int J Radiat Oncol Biol Phys. 1994; 29:1005. [PubMed: 8083069]

3. Gieschen HL, Spiro IJ, Suit HD, et al. Long-term results of intraoperative electron beam radiotherapy for primary and recurrent retroperitoneal soft tissue sarcoma. Int J Radiat Oncol Biol Phys. 2001; 50:127. [PubMed: 11316555]

4. Heslin MJ, Lewis JJ, Nadler E, et al. Prognostic factors associated with long-term survival for retroperitoneal sarcoma: implications for management. J Clin Oncol. 1997; 15:2832. [PubMed: 9256126]

5. Sindelar WF, Kinsella TJ, Chen PW, et al. Intraoperative radiotherapy in retroperitoneal sarcomas. Final results of a prospective, randomized, clinical trial. Arch Surg. 1993; 128:402. [PubMed: 8457152]

6. Nathan H, Raut CP, Thornton K, et al. Predictors of survival after resection of retroperitoneal sarcoma: a population-based analysis and critical appraisal of the AJCC staging system. Ann Surg. 2009; 250:970. http://dx.doi.org/10.1097/SLA.0b013e3181b25183. [PubMed: 19644351]

7. van Dalen T, Plooij JM, van Coevorden F, et al. Long-term prognosis of primary retroperitoneal soft tissue sarcoma. Eur J Surg Oncol. 2007; 33:234. http://dx.doi.org/10.1016/j.ejso.2006.09.020. [PubMed: 17081725]

8. Swallow CJ, Catton CN. Improving outcomes for retroperitoneal sarcomas: a work in progress. Surg Oncol Clin N Am. 2012; 21:317. [PubMed: 22365522]

9. Lewis JJ, Leung D, Woodruff JM, Brennan MF. Retroperitoneal soft-tissue sarcoma: analysis of 500 patients treated and followed at a single institution. Ann Surg. 1998; 228:355. [PubMed: 9742918]

10. Yoon SS, Chen YL, Kirsch DG, et al. Proton-beam, intensity-modulated, and/or intraoperative electron radiation therapy combined with aggressive anterior surgical resection for retroperitoneal sarcomas. Ann Surg Oncol. 2010; 17:1515. http://dx.doi.org/10.1245/s10434-010-0935-1. [PubMed: 20151216]

11. Yang JC, Chang AE, Baker AR, et al. Randomized prospective study of the benefit of adjuvant radiation therapy in the treatment of soft tissue sarcomas of the extremity. J Clin Oncol. 1998; 16:197. [PubMed: 9440743] 
12. Alvarenga JC, Ball AB, Fisher C, Fryatt I, Jones L, Thomas JM. Limitations of surgery in the treatment of retroperitoneal sarcoma. Br J Surg. 1991; 78:912. [PubMed: 1913104]

13. Jaques DP, Coit DG, Hajdu SI, Brennan MF. Management of primary and recurrent soft-tissue sarcoma of the retroperitoneum. Ann Surg. 1990; 212:51. [PubMed: 2363604]

14. Singer S, Corson JM, Demetri GD, Healey EA, Marcus K, Eberlein TJ. Prognostic factors predictive of survival for truncal and retroperitoneal soft-tissue sarcoma. Ann Surg. 1995; 221:185. [PubMed: 7857146]

15. Storm FK, Mahvi DM. Diagnosis and management of retroperitoneal soft-tissue sarcoma. Ann Surg. 1991; 214:2. [PubMed: 2064467]

16. Gilbeau L, Kantor G, Stoeckle E, et al. Surgical resection and radiotherapy for primary retroperitoneal soft tissue sarcoma. Radiother Oncol. 2002; 65:137. [PubMed: 12464441]

17. Alektiar KM, Hu K, Anderson L, Brennan MF, Harrison LB. High-dose-rate intraoperative radiation therapy (HDR-IORT) for retroperitoneal sarcomas. Int J Radiat Oncol Biol Phys. 2000; 47:157. [PubMed: 10758318]

18. Tran PT, Hara W, Su Z, et al. Intraoperative radiation therapy for locally advanced and recurrent soft-tissue sarcomas in adults. Int J Radiat Oncol Biol Phys. 2008; 72:1146. http://dx.doi.org/ 10.1016/j.ijrobp.2008.02.012. [PubMed: 18394818]

19. Sindelar WF, Kinsella T, Tepper J, Travis EL, Rosenberg SA, Glatstein E. Experimental and clinical studies with intraoperative radiotherapy. Surg Gynecol Obstet. 1983; 157:205. [PubMed: 6351303]

20. Kinsella TJ, DeLuca AM, Barnes M, Anderson W, Terrill R, Sindelar WF. Threshold dose for peripheral neuropathy following intraoperative radiotherapy (IORT) in a large animal model. Int J Radiat Oncol Biol Phys. 1991; 20:697. [PubMed: 2004946]

21. Sindelar WF, Kinsella TJ. Normal tissue tolerance to intraoperative radiotherapy. Surg Oncol Clin N Am. 2003; 12:925. [PubMed: 14989124]

22. Caudle AS, Tepper JE, Calvo BF, et al. Complications associated with neoadjuvant radiotherapy in the multidisciplinary treatment of retroperitoneal sarcomas. Ann Surg Oncol. 2007; 14:577. http:// dx.doi.org/10.1245/s10434-006-9248-9. [PubMed: 17119868]

23. Petersen IA, Haddock MG, Donohue JH, et al. Use of intraoperative electron beam radiotherapy in the management of retroperitoneal soft tissue sarcomas. Int J Radiat Oncol Biol Phys. 2002; 52:469. [PubMed: 11872294] 

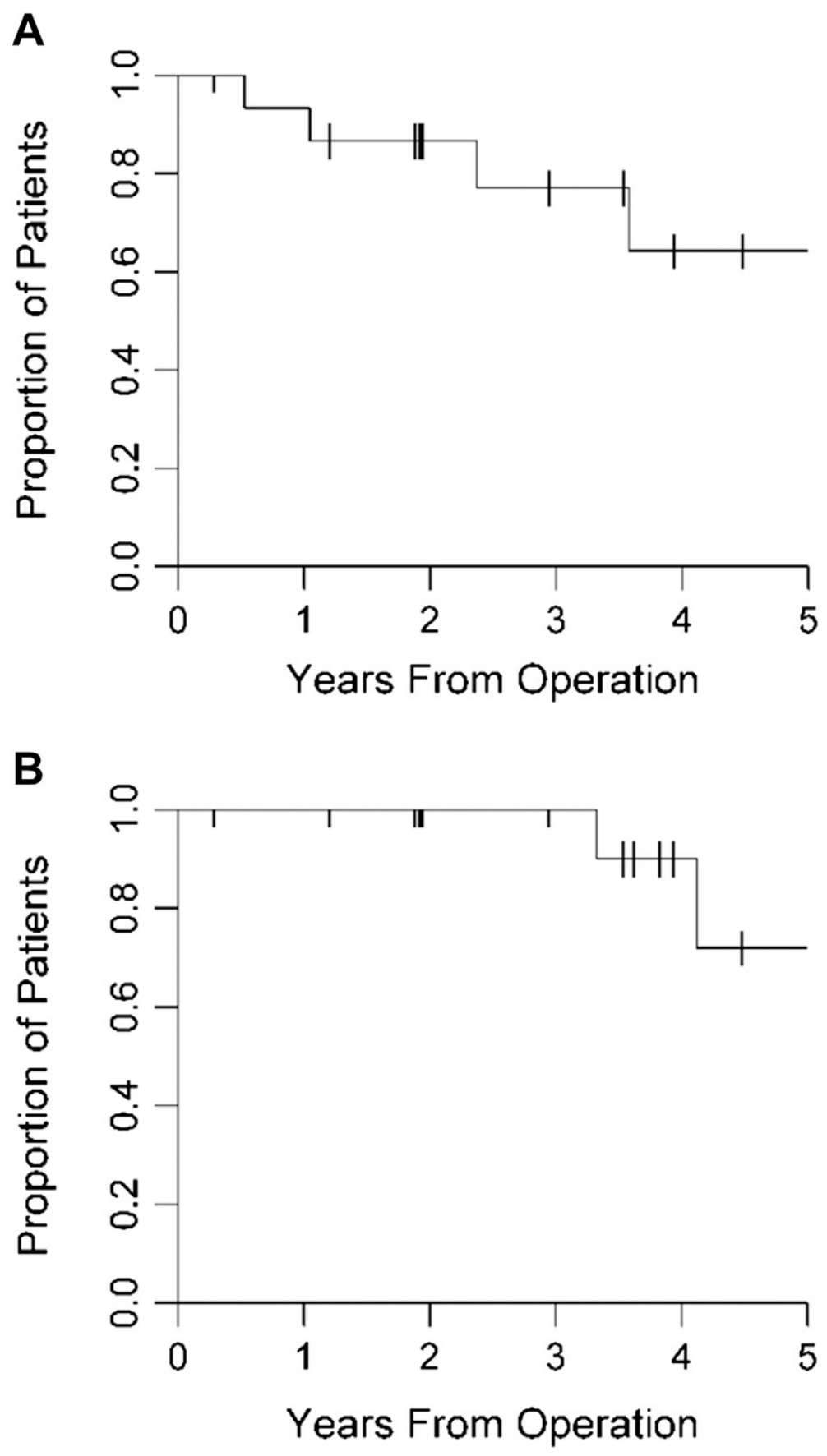

Fig.

(A) Time to LR was generated and analyzed from the date of diagnosis using the KaplanMeier method. The 5-y LR free rate for our patient population is 64\%. (B) OS was generated and analyzed from the date of diagnosis using the Kaplan-Meier method. The 2- and 5-y OS rates for our patient population are $100 \%$ and $72 \%$, respectively. 


\section{Table 1}

Demographics and tumor characteristics.

\begin{tabular}{|lc|}
\hline Variable & $N(\%)$ \\
\hline Total patients & 18 \\
Median follow-up & 43 mo (3-98) \\
Median age (range) & $51(25-76)$ \\
Gender & \\
Male & $9(50 \%)$ \\
Female & $9(50 \%)$ \\
Race & \\
White & $11(61 \%)$ \\
Black & $7(39 \%)$ \\
Presentation & \\
Primary disease & $13(72 \%)$ \\
Recurrence disease & $5(28 \%)$ \\
Histology & \\
Liposarcoma & $9(50 \%)$ \\
Leiomyosarcoma & $5(28 \%)$ \\
MFH & $2(11 \%)$ \\
Other & $2(11 \%)$ \\
Grade & $16(89 \%)$ \\
Low (1, 2) & $2(11 \%)$ \\
High (3, 4) & $15(3-41)$ \\
Margin status & \\
R0 & \\
R1 & \\
Median tumor size (cm) & \\
& \\
&
\end{tabular}

MFH $=$ Malignant Fibrous Histiocytoma. 


\section{Table 2}

Local recurrence.

\begin{tabular}{|lcrc|}
\hline Variable & LR & $\boldsymbol{N}$ & LR rate $(\%)$ \\
\hline All patients & 5 & 16 & 31 \\
Primary disease & 3 & 12 & 25 \\
Recurrent disease & 2 & 4 & 50 \\
High grade & 4 & 7 & 57 \\
Low grade & 1 & 9 & 11 \\
\hline
\end{tabular}




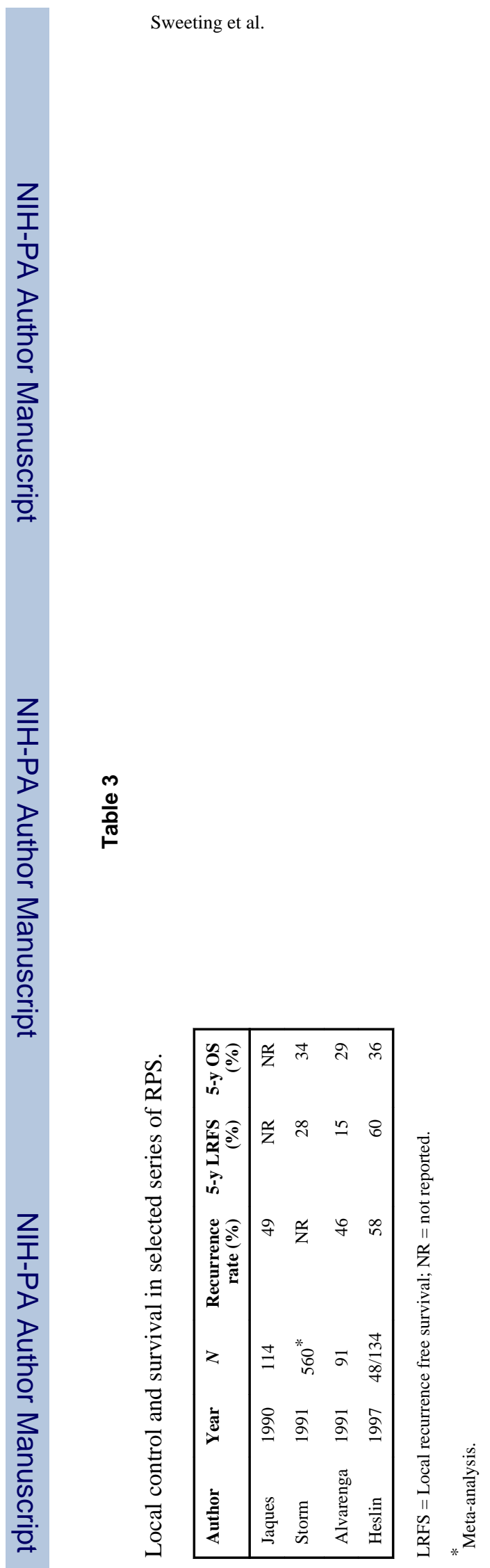

J Surg Res. Author manuscript; available in PMC 2014 September 18. 
Table 4

Local control and survival with use of IOERT in RPS.

\begin{tabular}{|lcccc|}
\hline Author & Year & $\boldsymbol{N}$ & 5-y LRFS $(\boldsymbol{\%})$ & 5-y OS $(\boldsymbol{\%})$ \\
\hline Sindelar & 1993 & 15 & $31^{*}$ & $37^{*}$ \\
Alektiar & 2000 & 32 & 62 & 45 \\
Geischen & 2001 & 16 & 83 & 74 \\
Tran & 2008 & 39 & 24 & $30^{\dagger}$ \\
Yoon & 2010 & 12 & 60 & 36 \\
Our study & & 18 & 64 & 72 \\
\hline
\end{tabular}

* Estimated from survival curves.

${ }^{\dagger}$ Disease-specific survival. 